\title{
Tumor necrosis factor-related apoptosis-inducing ligand induces cytotoxicity specific to osteosarcoma by microRNA response elements
}

\author{
FEI XIAO, JUWU CHEN, CHUANJU LIAN, PENGCHAO HAN and CHAOYANG ZHANG \\ Department of Emergency Surgery, The First Affiliated Hospital of Zhengzhou University, \\ Zhengzhou, Henan 450052, P.R. China
}

Received February 17, 2014; Accepted September 9, 2014

DOI: $10.3892 / \mathrm{mmr} .2014 .2710$

\begin{abstract}
As the most common primary bone neoplasm, osteosarcoma is highly aggressive and represents a high risk to human health. Biological agents, including tumor necrosis factor-related apoptosis-inducing ligand (TRAIL), are considered promising therapeutic strategies for osteosarcoma. The current issue limiting the application of TRAIL gene therapy is that normal cells are also affected due to the lack of tumor selectivity. The present study aimed to employ the miRNA response elements (MREs) of microRNA ( $m i R)-34$ and $m i R-122$, which are tumor suppressors, to enable the selective expression of TRAIL by adenoviral vectors in osteosarcoma cells. The results revealed that $m i R-34$ and $m i R-122$ were underexpressed in osteosarcoma tissues, compared with normal tissues. The MREs of $m i R-34$ and $m i R-122$ ensured that the luciferase gene was expressed selectively in osteosarcoma cells. Adenovirus (Ad)-TRAIL-34-122, which expressed TRAIL in an $m i R-34$ and $m i R-122$-regulated manner, selectively expressed TRAIL in the osteosarcoma cells assessed, which was detected using reverse transcription quantitative polymerase chain reaction, immunoblotting and ELISA. Apoptosis and cytotoxicity were also detected in the osteosarcoma cells, compared with the normal cells. Animal experiments further indicated that Ad-TRAIL-34-122 was able to reduce the growth of osteosarcoma xenografts without toxicity to the liver. In conclusion, the present study identified a novel miRNA-regulated biological cancer therapy against osteosarcoma, which is tumor selective and may be promising for future clinical treatment.
\end{abstract}

Correspondence to: Dr Fei Xiao, Department of Emergency Surgery, The First Affiliated Hospital of Zhengzhou University, 1 Erqijianshe East Road, Zhengzhou, Henan 450052, P.R. China E-mail: feixiaozhengzhou@gmail.com

Key words: osteosarcoma, adenovirus, microRNA-34, microRNA-122, tumor necrosis factor-related apoptosis-inducing ligand

\section{Introduction}

Osteosarcoma is one of the most frequent types of malignant cancer and poses a threat to human health (1). It has been revealed that the aberrant expression of tumor suppressor genes is important in the progression, invasion and metastasis of osteosarcoma (1). Thus, gene therapy is an effective strategy for the treatment of osteosarcoma (2).

Tumor necrosis factor-related apoptosis-inducing ligand (TRAIL) can induce apoptosis in various cancer cells by binding cellular receptors, death receptor (DR)4 and DR5, and activating the downstream signal transduction pathway $(3,4)$. Adenovirus (Ad)-mediated TRAIL delivery has been well documented to exert antitumor activity against osteosarcoma, both in vitro and in animal models $(5,6)$. Furthermore, the extensive expression of DR4 and DR5 in osteosarcoma ensures that TRAIL may be an effective strategy in future clinical treatment (7).

However, TRAIL has been demonstrated to induce apoptosis in hepatic cells by inducing the FAS-mediated downstream molecular pathway (8). The cytotoxicity of TRAIL to normal liver cells impedes its clinical application in the treatment of osteosarcoma. Therefore, a novel gene therapy strategy, which is able to protect normal tissue, particularly liver tissue, from the side effects of TRAIL expression is required.

Differential microRNA (miRNA) expression levels have been revealed between osteosarcoma and normal cells (9). A decrease in the expression of certain miRNAs enables the selective expression of inserted genes in osteosarcoma cells by their miRNA response elements (MREs). For example, $m i R-34$ has been demonstrated to be underexpressed in osteosarcoma cells (10) and $m i R-122$ is as a liver-enriched miRNA that can be used to suppress the expression of TRAIL in liver cells and thus minimize hepatotoxicity (11). However, to the best of our knowledge, the effectiveness of MRE-based gene therapy for the treatment of osteosarcoma has not yet been investigated.

In the present study, MREs of $m i R-34$ and $m i R-122$ were used to regulate the expression of TRAIL in order to confer its expression with osteosarcoma selectivity. Multidisciplinary experiments were performed to verify its effectiveness. 


\section{Materials and methods}

Ad construction. Ad-enhanced green fluorescent protein (EGFP) and Ad-TRAIL were provided by Dr Zhao (Department of Urology, General Hospital of Chengdu Military Area Command of Chinese PLA, Chengdu, China). Ad-TRAIL-34-122 was constructed as follows: A DNA fragment containing two copies of the MREs of $m i R-34$ and $m i R$-122 (5'-GCCGATATCACAAACACCCACTGCCACAA CACCCACTGCCACAAACACCACACTCCACAAACCACAC TCCGATATCGGC-3') was synthesized and released from the temporary vector by EcoRV. The fragment was then inserted into pShuttle-cytomegalovirus (CMV)-TRAIL at the same site to obtain pShuttle-CMV-TRAIL-34-122. Subsequently, pShuttle-CMV-TRAIL-34-122 and pAdEasy were cotransfected into human embryonic kidney (HEK)293 cells (Microbix Biosystems, Mississauga, ON, Canada). The adenoviral vectors were purified using a $\mathrm{CsCl}$ centrifugation method $(30,000 \times \mathrm{g}, 2.5 \mathrm{~h})$, following identification by reverse transcription quantitative polymerase chain reaction (RT-qPCR). The titers of Ad-EGFP, Ad-TRAIL-34-122 and Ad-TRAIL were determined using a TCID $_{50}$ method on HEK293 cells and presented as $\mathrm{pfu} / \mathrm{ml}$.

Cell line cultures. The human osteosarcoma cell lines, KHOS, HOS, SaOS2, U2OS and MG-63 and the human normal lung fibroblast cell lines, NHLF and MRC5, were all purchased from the American Type Culture Collection (ATCC; Manassas, VA, USA). Normal liver cells, L-02, were obtained from Shanghai Cell Collection (Shanghai, China). The cells were cultured using the recommended media (ATCC-formulated Eagle's minimum essential medium for KHOS, HOS, MG-63, NHLF, and MRC5; ATCC-formulated McCoy's 5a medium modified for Saos-2; Dulbecco's modified Eagle's minimum essential medium for U2OS, and HEK-293; RPMI-1640 medium for L-02 (Invitrogen, Grand Island, NY, USA) supplemented with $10 \%$ fetal bovine serum, $4 \mathrm{mM}$ glutamine, $100 \mathrm{U} / \mathrm{ml}$ penicillin and $100 \mu \mathrm{g} / \mathrm{ml}$ streptomycin (all Invitrogen) at $37^{\circ} \mathrm{C}$ and $5 \%$ $\mathrm{CO}_{2}$ in a humidified atmosphere.

$R T-q P C R$. For the detection of $m i R-34$ and $m i R-122$, fresh osteosarcoma samples were obtained from surgery (Department of Emergency Surgery, The First Affiliated Hospital of Zhengzhou University, Zhengzhou, China). Total RNA was extracted from 10 cancerous samples and corresponding normal tissues using TRIzol solution (Sigma-Aldrich, St. Louis, MO, USA) and pooled as one group for subsequent experiments. In addition, the KHOS, HOS, SaOS2, U2OS, MG-63, NHLF, MRC5 and L-02 cells were subjected to RNA extraction using TRIzol. A TaqMan ${ }^{\circledR}$ MicroRNA Reverse Transcription kit (Applied Biosystems, Foster City, CA, USA) was applied to perform reverse transcription, followed by TaqMan ${ }^{\circledR} 2 \mathrm{X}$ Universal PCR Master mix kit (Applied Biosystems)-based qPCR assays using a CFX96 ${ }^{\text {TM }}$ Real-Time PCR Detection System (Bio-Rad Laboratories, Hercules, CA, USA). The data were processed and analyzed using REST 2009 software (Qiagen, Hilden, Germany).

The procedure used to detect TRAIL mRNA in the Ad-TRAIL-34-122-infected cells was as follows: In each well of 24 -well plates, $4 \times 10^{4}$ cells were seeded. These cells were infected with Ad-TRAIL or AD-TRAIL-34-122 with a multiplicity of infection (MOI) of 10. After $48 \mathrm{~h}$, total RNA was extracted using TRIzol ${ }^{\circledR}$ solution (Invitrogen Life Technologies, Carlsbad, CA, USA), followed by cDNA generation by reverse transcription using a ReverTra Ace qPCR RT kit (Toyobo Co., Ltd., Osaka, Japan) according to the manufacturer's instructions. Subsequently, SYBR premix Ex Taq (Takara Bio, Inc., Otsu, Japan) was used for RT-qPCR. The primers used for TRAIL detection were as follows: TRAIL, forward: 5'-GACCTGCGTGCTGATC-3' and reverse: 5'-TAAAAGAAGATGACAG-3'.

Luciferase assay. A DNA fragment, containing two copies of the miR-34 and miR-122 MREs (5'-GCCCTCGAGACAACCAGCTAAGACACTGCCAACAACCAGCTAAGA-

CACTGC

CACAAACACCATTGTCACACTCCACAAACACCATTGT

CACACTCCAGCGGCCGCGGC-3'), was synthesized and inserted into a psiCheck2 vector at the sites XhoI and Not I (Promega Corporation, Madison, WI, USA) in order to generate psiCheck2-34-122.

Cells $\left(4 \times 10^{4}\right)$ were cultured in each well of a 24 -well plate. psiCheck2-34-122 and psiCheck2 were transfected into the cells using Lipofectamine 2000 (Invitrogen Life Technologies) and, $48 \mathrm{~h}$ later, these cells were treated with lysis buffer (Promega Corporation). The luciferase activities were then evaluated using a Dual-Luciferase reporter system kit (Promega Corporation).

Immunoblot assay. The total proteins were extracted using an M-PER ${ }^{\circledR}$ Mammalian Protein Extraction reagent (Thermo Fisher Scientific, Rockford, IL, USA) and then separated on $10 \%$ SDS-PAGE gels (Thermo Fisher Scientific). Subsequently, the proteins were transferred onto $0.45 \mu \mathrm{m}$ nitrocellulose membranes (Thermo Fisher Scientific) and 5\% fat-free dry milk was added to block the membrane. After $1 \mathrm{~h}$, the membranes were incubated with the primary antibodies for $12 \mathrm{~h}$ at $4^{\circ} \mathrm{C}$ : Monoclonal rabbit anti-TRAIL, monoclonal rabbit anti-cle-PARP, monoclonal rabbit anti-cle-caspase 3, and monoclonal mouse anti-GAPDH. On day 2 , the membranes were then incubated with the matched polyclonal goat anti-mouse or anti-rabbit immunoglobulin $\mathrm{G}$ secondary antibodies for $1 \mathrm{~h}$ at room temperature and the blots were detected using SuperSignal West Dura Extended Duration substrate (Thermo Fisher Scientific). All the above antibodies were all purchased from Cell Signaling Technology (Boston, MA, USA).

TRAIL determination by ELISA. To determine the concentration of secreted TRAIL, ELISA was performed. A total of $3.5 \times 10^{5}$ cells were cultured in each well of a 6 -well plate and different adenoviral vectors (10 MOI) were added. After $48 \mathrm{~h}$, sandwich ELISA (Thermo Fisher Scientific) was used to detect the expression level of TRAIL secreted into the media. The following antibodies were used: Monoclonal mouse TRAIL antibody and polyclonal goat TRAIL antibody (R\&D Systems, Inc.) Absorbance was measured at a wavelength of $450 \mathrm{~nm}$ using a Microplate Reader (model 550; Bio-Rad Laboratories).

miRNA mimics and inhibitors. The mimics and inhibitors for $m i R-34$ and $m i R-122$ as well as the control mimics and 
inhibitors were purchased from GenePharma (Shanghai, China). Prior to subsequent experiments, the indicated cells were transfected with the control mimic $(200 \mathrm{nM})$ or a mixture of the $m i R-34$ mimic $(100 \mathrm{nM})$ and the $m i R-122$ $\operatorname{mimic}(100 \mathrm{nM})$.

Determination of apoptotic rates. The cells $\left(2 \times 10^{5}\right)$ were cultured in 6-well plates. After $24 \mathrm{~h}$, the indicated adenoviruses (10 MOI) were added to the media and, after $48 \mathrm{~h}$, the cells were processed using an Annexin V-PE Apoptosis Detection kit (BioVision, Inc., Milpitas, CA, USA) according to the manufacturer's instructions. The apoptotic rate was then determined using flow cytometry.

Cell viability assay. The cells $\left(1 \times 10^{4}\right)$ were cultured in each well of a 96-well plate. The cells were infected with the indicated adenoviral vector of different MOI. After 6 days, $50 \mu \mathrm{l}$ dimethyl sulfoxide (DMSO) was added to the cell media for $4 \mathrm{~h}$ incubation, and then the media was discarded and $150 \mu \mathrm{l}$ DMSO was added. The $570 \mathrm{~nm}$ absorbance in each well was then assessed using a microplate reader (Model 550; Bio-Rad Laboratories) with a reference wavelength of $655 \mathrm{~nm}$. Cell viability was calculated using the following formula: Cell viability $(\%)=$ absorbance value of infected cells - background absorbance / absorbance of uninfected control cells - background absorbance.

Animal experiments. The protocols of animal experiments were approved by the Experimental Animal Ethics Committee of Zhengzhou University.

To establish osteosarcoma xenografts, $2 \times 10^{6}$ KHOS cells were injected into the left flanks of 5-week-old BALB/c nude mice. A total of 24 mice were divided equally into four groups $(n=6)$, once the tumor diameter reached 7-9 mm. Needles were used to intratumorally inject $100 \mu \mathrm{l}$ PBS either with or without $2 \times 10^{8}$ pfu Ad-EGFP, Ad-TRAIL or Ad-TRAIL-34-122 five times every other day. The total dosage of adenoviruses reached $1 \times 10^{9} \mathrm{pfu}$. Calipers were used to periodically measure the tumor diameters. Tumor volume was then calculated using the following formula: Tumor volume $\left(\mathrm{mm}^{3}\right)=$ maximal length $(\mathrm{mm}) \mathrm{x}$ perpendicular width $(\mathrm{mm})^{2} / 2$.

Liver function evaluation. Female BALB/c mice $(n=5)$ were intravenously administered with Ad-EGFP, Ad-TRAIL or Ad-TRAIL-34-122 (1x10 $\left.10^{9} \mathrm{pfu}\right)$ five times every other day, in order to determine the resulting hepatotoxicity. After 10 days, $600 \mathrm{ml}$ serum was obtained from each mouse by cardiac puncture and then incubated with heparin (12 U). Serum alanine aminotransferase (ALT) was subsequently quantified at the Clinical Laboratory, The First Affiliated Hospital of Zhengzhou University.

Histological staining. A single mouse from each group was sacrificed 7 days after adenovirus administration. The tumor and liver tissues were fixed using formalin followed by histological staining based on the streptavidin biotin peroxidase complex method. TRAIL protein expression and distribution were determined using the TRAIL antibody (Santa Cruz Biotechnology, Inc., Santa Cruz, CA, USA). Prior to observation, the sections were counterstained with hematoxylin.
Statistical analysis. A two-tailed Student's t-test was used for statistical analysis in the present study. $\mathrm{P}<0.05$ was considered to indicate a statistically significant difference.

\section{Results}

Levels of miR-34 are reduced in osteosarcoma. To confirm the downregulation of $m i R-34$ in osteosarcoma, the miRNA expression profiles were examined in 10 samples of osteosarcoma. RT-qPCR indicated that the expression levels of $m i R-34$ were decreased in osteosarcoma, compared with the corresponding normal tissue $(\mathrm{P}<0.01$; Fig. 1A). Furthermore, the abundance of miR-34 was quantified in several osteosarcoma cell lines and levels were also found to be reduced in the cell lines assessed (Fig. 1B). The differential expression profile of $m i R$-34 suggested that the application of these miRNAs regulated the expression of exogenous genes in the osteosarcoma cells.

Application of miR-34 and miR-122 MREs regulates the expression of TRAIL in an osteosarcoma-selective manner. To investigate whether the MREs of $m i R-34$ and $m i R-122$ can be applied for osteosarcoma-specific expression of exogenous genes, psiCkeck2-34-122 U, a luciferase reporter vector regulated by the MREs of $m i R-34$ and $m i R-122$, was constructed (Fig. 1C). The results demonstrated that luciferase activity was not significantly affected in the psiCkeck2-34-122-transfected osteosarcoma cells. However, its activity was markedly suppressed in the normal cell lines (Fig. 1D).

MREs of miR-34 and miR-122 restrict the expression of TRAIL mediated by adenoviral vectors within osteosarcoma cells. To confirm whether the application of $m i R-34$ and $m i R-122$ MREs enabled the selective expression of TRAIL in osteosarcoma cells, an adenoviral vector was constructed by inserting two copies of the miR-34 and miR-122 MREs at the end of the TRAIL-encoding sequence (Fig. 2A). The RT-qPCR assay revealed no difference in the expression of TRAIL between the Ad-TRAIL-34-122 and Ad-TRAIL-infected osteosarcoma cells (Fig. 2B). By contrast, Ad-TRAIL-34-122 suppressed the expression of TRAIL protein in normal cells (Fig. 2B). Immunoblotting and ELISA further demonstrated that Ad-TRAIL-34-122 treatment led to the selective expression of TRAIL in osteosarcoma (Fig. 2C and D). To verify that TRAIL expression was regulated by the abundance of endogenous $m i R-34$ and $m i R-122$, the inhibitors or mimics of these miRNAs were added to the cell cultures. The data demonstrated that increasing the abundance of $m i R-34$ and $m i R-122$ in osteosarcoma cells led to a reduction in the expression of TRAIL proteins, while suppression of $m i R-34$ and $m i R-122$ by inhibitors resulted in the restoration of TRAIL in normal cells (Fig. 2E).

Selective apoptosis in osteosarcoma cells is induced by miRNA-regulated expression of TRAIL. As TRAIL initiates apoptosis in a wide range of cells, the present study investigated the activation of the apoptotic pathway in osteosarcoma and normal cell lines. Flow cytometric analysis of apoptotic rates revealed that apoptosis occurred specifically in the osteosarcoma cells, but not in the normal cells, infected with 
A

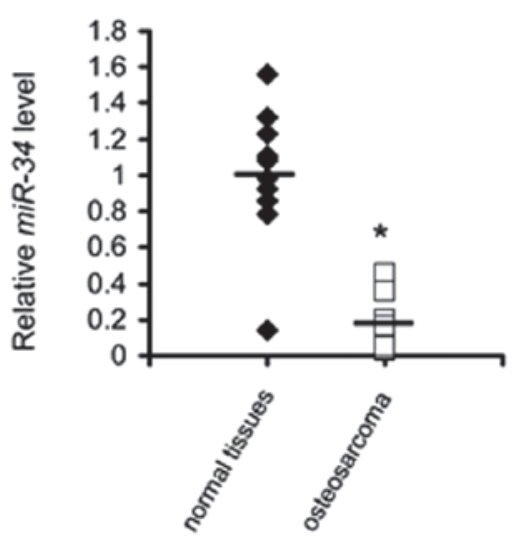

B

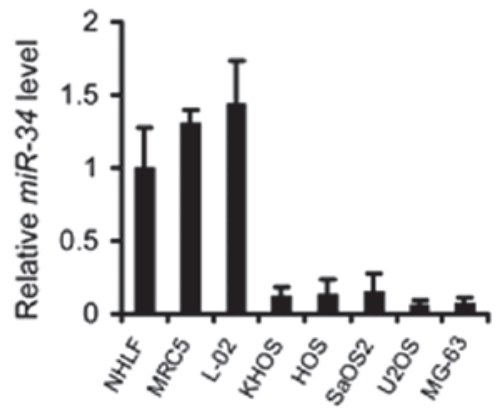

C

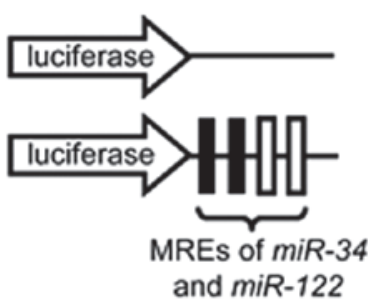

D

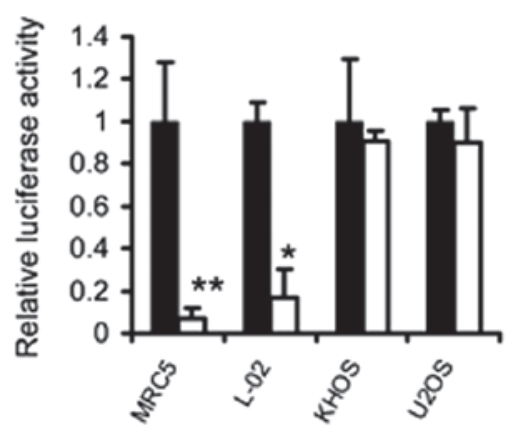

Figure 1. Exogenous genes are selectively expressed in osteosarcoma cells mediated by the MREs of $m i R-34$ and $m i R-122$. (A) Expression of $m i R-34$ and $m i R-122$ was detected in the osteosarcoma specimens and their corresponding normal tissues $(n=10)$. Data are expressed as the mean \pm standard error of the mean of three independent experiments. (B) Expression of $m i R-34$ and $m i R-122$ was detected in NHLF, MRC-5, L-02, KHOS, HOS, SaOS2, U2OS and MG-63 cells. Data are expressed as the mean \pm standard error of the mean of three independent experiments. (C) Structures of the reporter vectors. (D) Luciferase activity was quantified in the MRC-5, L-02, KHOS and U2OS cells transfected with the control vector psiCheck2 (ש) or psiCheck2-34-122 ( $\square$ ). Data are expressed as the mean \pm standard error of the mean of three independent experiments. ${ }^{*} \mathrm{P}<0.05,{ }^{* *} \mathrm{P}<0.01$. miR, microRNA; MREs, miRNA response elements.
Ad-TRAIL-34-122 (Fig. 3A). By contrast, Ad-TRAIL induced apoptosis in osteosarcoma and normal cells (Fig. 3A).

Furthermore, immunoblot assays were also employed to confirm the activation status of the apoptotic pathway in osteosarcoma cells infected with TRAIL-expressing adenoviruses. Caspase- 3 and poly ADP-ribose polymerase were found to be markedly cleaved in the Ad-TRAIL and Ad-TRAIL-34-122-transduced osteosarcoma cells and in the Ad-TRAIL-infected normal cells. However, no cleavage was observed in the normal cells infected with Ad-TRAIL-34-122 (Fig. 3B).

Ad-TRAIL-34-122 compromises the viability of osteosarcoma cells without significant cytotoxicity to normal cells. MTT assays were subsequently performed to determine the suppressive effect of Ad-TRAIL and Ad-TRAIL-34-122 on osteosarcoma cells and normal cells. According to the data, the viability of the osteosarcoma cells was inhibited when Ad-TRAIL and Ad-TRAIL-34-122 were added to the cell culture (Fig. 4A and B). However, the effect of Ad-TRAIL and Ad-TRAIL-34-122 on normal cells differed. Ad-TRAIL reduced the survival of normal cell lines, whereas their viability was not affected by Ad-TRAIL-34-122 (Fig. 4C and D).

TRAIL expression suppresses the growth of osteosarcomaxenografts in vivo. Subsequently, the effect of Ad-TRAIL-34-122 on the growth of osteosarcoma xenografts in mice was investigated. KHOS cells were used to establish the osteosarcoma xenografts. Mice were then intravenously injected with PBS, Ad-EGFP, Ad-TRAIL and Ad-TRAIL-34-122 and the tumor diameter was periodically measured. The data revealed that Ad-TRAIL and Ad-TRAIL-34-122 suppressed KHOS tumor growth in mice (Fig. 5A). The expression of TRAIL was also confirmed in the tumor sections using immunohistological staining (Fig. 5B).

Ad-TRAIL-34-122 exhibits no hepatotoxicity in mice. Finally, the present study investigated whether these adenoviral vectors led to hepatotoxicity in mice. PBS, Ad-EGFP, Ad-TRAIL and Ad-TRAIL-34-122 were injected into the tail vein of tumor-free BALB/c mice. The blood was harvested for analysis of serum ALT levels. The results revealed that Ad-TRAIL induced significant hepatotoxicity, evidenced by the elevated levels of ALT in the blood. However, Ad-TRAIL-MRE-34-122 did not alter the levels of ALT in the blood (Fig. 5C). Furthermore, immuohistological staining indicated that TRAIL was not expressed in the liver tissues from the mice injected with Ad-TRAIL-34-122 (Fig. 5B).

\section{Discussion}

The present study initially verified that the expression of $m i R-34$ and $m i R-122$ was underexpressed in osteosarcoma compared with in normal tissues. These results were consistent with a previous study (10). The difference in the levels of $m i R-34$ and $m i R-122$ between cancer and normal tissues suggested that the application of their MREs may confer the expression of inserted genes with specificity.

Further investigation using a luciferase reporter revealed that the MREs of $m i R-34$ and $m i R-122$ were able to suppress 
A

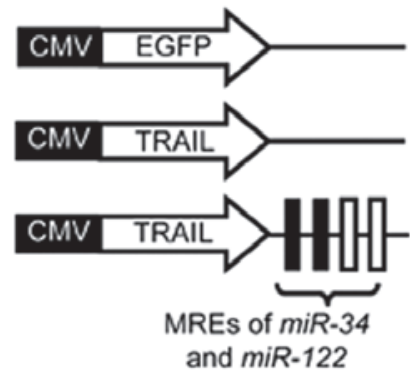

B

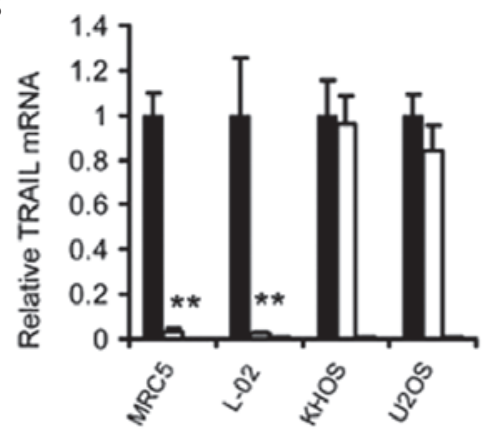

C

L-02

KHOS

TRAIL

GAPDH
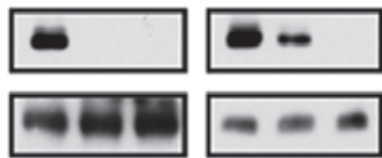

Ad-TRAIL $+-\quad+-$

Ad-TRAIL-34-122 - + - ++

Ad-EGFP
D

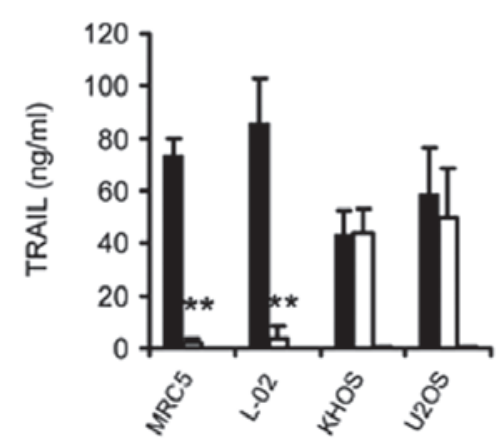

E

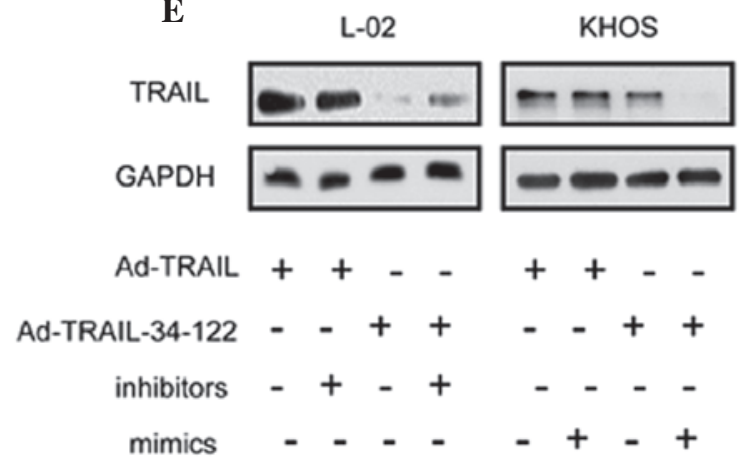

Figure 2. MREs of miR-34 and miR-122 confer TRAIL expression with osteosarcoma specificity. (A) Structures of the adenoviral vectors used in the present study. (B) Reverse transcription quantitative polymerase chain reaction was performed to detect TRAIL mRNA expression following infection of the cells with adenoviruses of 10 MOI. Ad-TRAIL (®); Ad-TRAIL-34-122 (). Data are expressed as the mean \pm standard error of the mean of three independent experiments. (C) TRAIL protein level was determined in the cells infected with different adenoviruses using immunoblot assays. GAPDH was selected as an endogenous reference. (D) TRAIL protein level was evaluated in the same cells infected with the indicated adenoviruses using ELISA. Ad-TRAIL (汭; Ad-TRAIL-34-122 (). Data are expressed as the mean \pm standard error of the mean of three independent experiments. (E) L-02 and KHOS cells were treated with Ad-TRAIL or Ad-TRAIL-34-218 of $10 \mathrm{MOI}$ and with mixed inhibitors or mimics of $m i R-34$ and $m i R-122$ (100 nM each). After $48 \mathrm{~h}$, the expression of TRAIL was assessed using an immunoblot assay. GAPDH was selected as an endogenous reference. miR, microRNA; MRE, miRNA response elements; TRAIL, tumor necrosis factor-related apoptosis-inducing ligand; Ad, adenovirus; CMV, cytomegalovirus; EGFP, enhanced green fluorescent protein; MOI, multiplicity of infection.
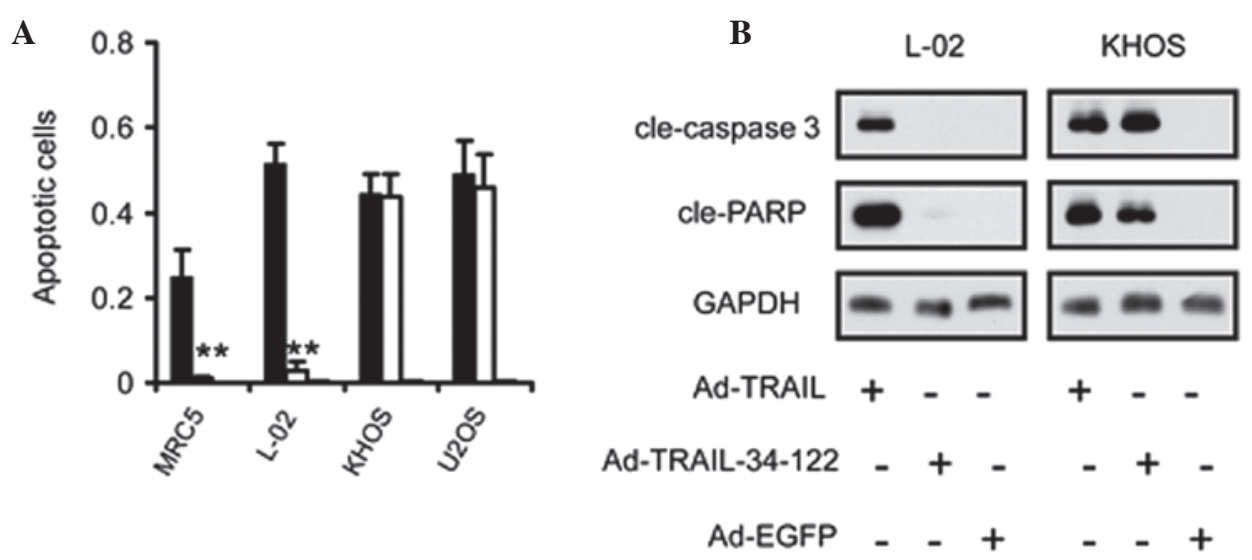

Figure 3. Selective activation of the apoptotic pathway is induced by Ad-TRAIL-34-122 infection. (A) Apoptosis was detected in the indicated cells using flow

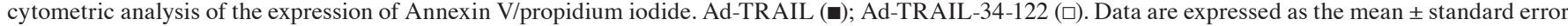
of the mean of three independent experiments. (B) Cleavage of caspase-3 and PARP were determined using an immunoblot assay. GAPDH was selected as an endogenous reference. Ad, adenovirus; TRAIL, tumor necrosis factor-related apoptosis-inducing ligand; PARP, poly ADP-ribose polymerase; EGFP, enhanced green fluorescent protein.

exogenous gene expression in the normal cells rather than in the osteosarcoma cells. This effect of MREs on the expression of inserted genes has been confirmed in other types of cancer $(12,13)$.
As expected, Ad-TRAIL-34-122 suppressed the viability of osteosarcoma cells by activating the apoptotic pathway and had no significant effect on the survival of normal cells, indicating high tumor selectivity. The use of MREs of tumor suppressor 

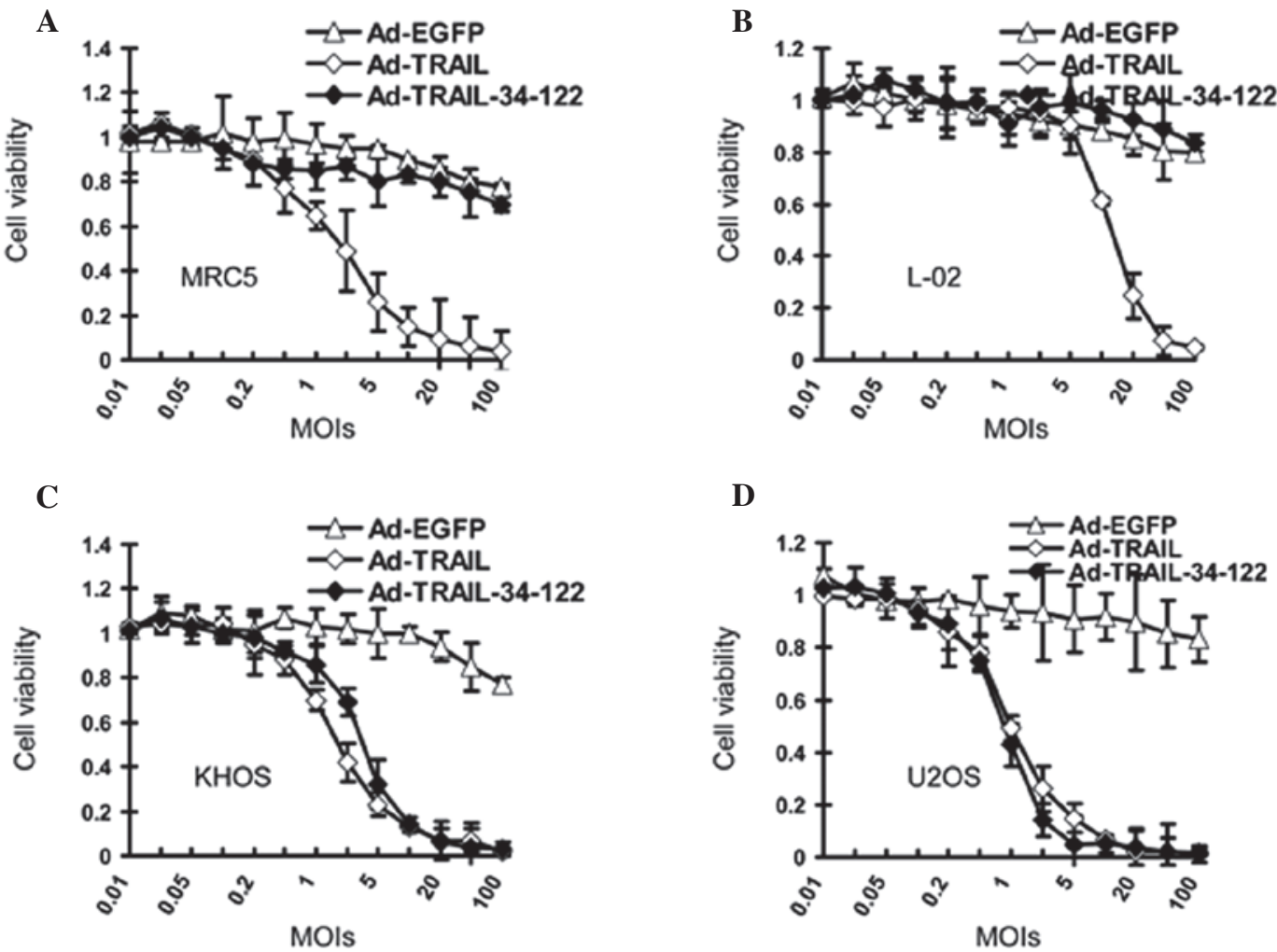

Figure 4. Ad-TRAIL-34-122 reduces the viability of osteosarcoma without significant cytotoxicity to normal cells. Viability was determined in (A) MRC5, (B) L-02, (C) KHOS and (D) U2OS cells following application of the indicated adenoviruses. The absorption values of the cells without adenovirus infection were used as standards. Data are expressed as the mean \pm standard error of the mean of three independent experiments. Ad, adenovirus; TRAIL, tumor necrosis factor-related apoptosis-inducing ligand; EGFP, enhanced green fluorescent protein; MOI, multiplicity of infection.
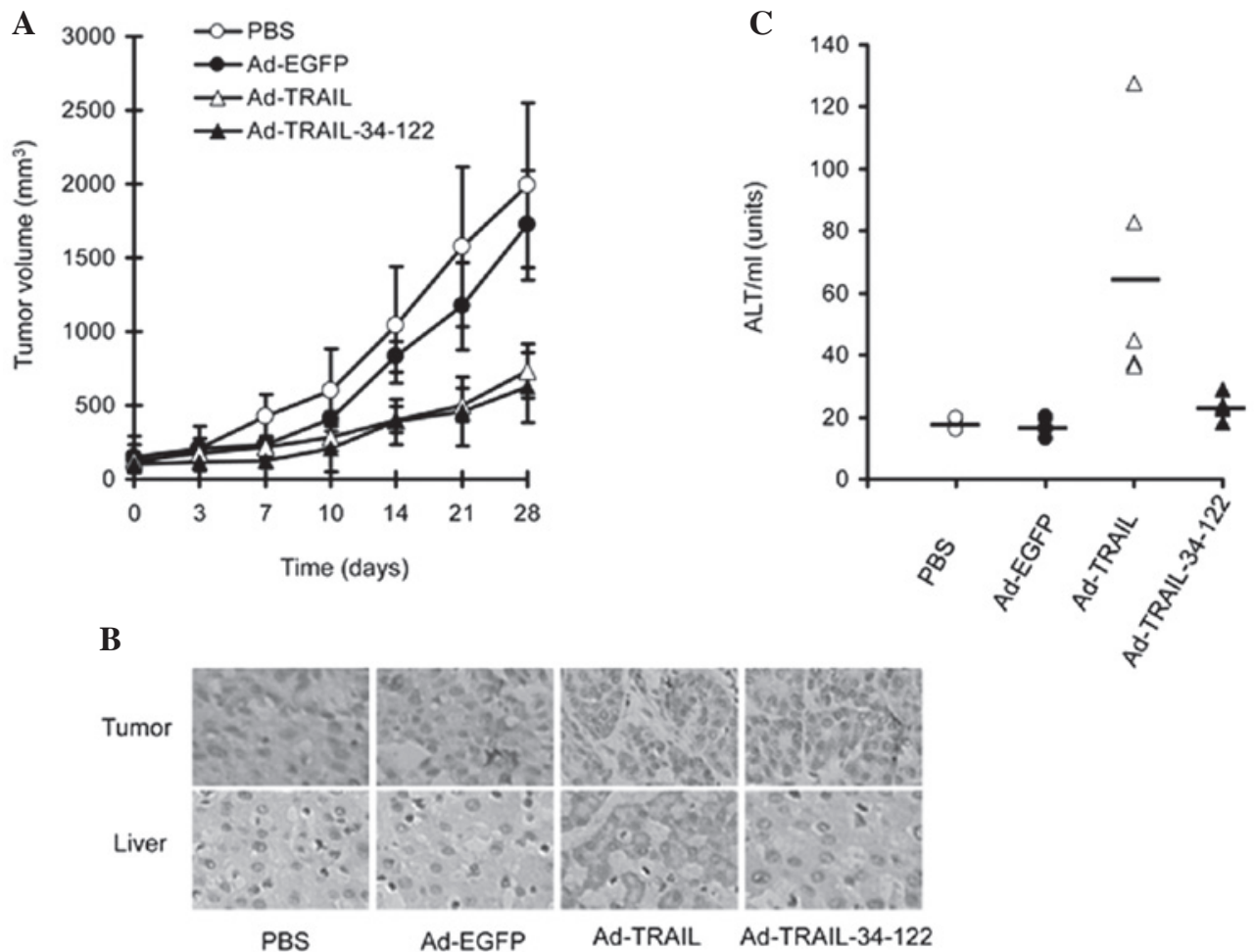

Figure 5. Ad-TRAIL-34-122 suppresses the growth of osteosarcoma xenografts in mouse models without hepatotoxicity. (A) Different adenoviruses (1x $10^{9}$ pfu) were intratumorally injected into mice bearing KHOS tumors and the tumor volumes were periodically measured. Data are expressed as the mean \pm standard error of the mean of tumor sizes. (B) Histological staining was performed to detect the expression of TRAIL in tumor and liver sections from the KHOS tumor-bearing mice and the tumor-free mice, respectively, following treatment with PBS, Ad-EGFP, Ad-TRAIL and Ad-TRAIL-34-122. Representative images are presented (magnification, x200). (C) ALT levels were detected in mice bearing no tumors following injection of different adenoviruses. Data are expressed as the mean \pm standard error of the mean of ALT serum levels. PBS, phosphate-buffered saline; Ad, adenovirus; TRAIL, tumor necrosis factor-related apoptosis-inducing ligand; EGFP, enhanced green fluorescent protein; ALT, alanine aminotransferase. 
miRNAs, including TRAIL, to regulate exogenous expression, mediated by adenoviral vectors, has been assessed in bladder cancer and glioma $(13,14)$. The therapeutic effect has been further verified in mice (13.14).

In addition to TRAIL, certain cytokines can also be used in MRE-regulated gene therapy for osteosarcoma, including dickkopf-1 and interleukin-24 $(15,16)$. Furthermore, adenoviral vectors from other serotypes are also suitable for MRE-regulated TRAIL therapy $(15,17)$ due to their improved infectivity towards cancer cells.

In the present study, an MRE-regulated adenoviral vector that selectively expressed TRAIL in osteosarcoma cells was constructed. The results confirmed that this strategy was effective and safe and demonstrated that this miRNA-based anti-tumor gene therapy may be promising for the treatment of osteosarcoma.

\section{References}

1. Ando K, Heymann MF, Stresing V, Mori K, Redini F and Heymann D: Current therapeutic strategies and novel approaches in osteosarcoma. Cancers (Basel) 5: 591-616, 2013.

2. Yang J and Zhang W: New molecular insights into osteosarcoma targeted therapy. Curr Opin Oncol 25: 398-406, 2013.

3. Walczak H, Miller RE, Ariail K, et al: Tumoricidal activity of tumor necrosis factor-related apoptosis-inducing ligand in vivo. Nat Med 5: 157-163, 1999.

4. Muzio M, Chinnaiyan AM, Kischkel FC, et al: FLICE, a novel FADD-homologous ICE/CED-3-like protease, is recruited to the CD95 (Fas/APO-1) death - inducing signaling complex. Cell 85: 817-827, 1996

5. Li C, Cheng Q, Liu J, Wang B, Chen D and Liu Y: Potent growth-inhibitory effect of TRAIL therapy mediated by double-regulated oncolytic adenovirus on osteosarcoma. Mol Cell Biochem 364: 337-344, 2012.

6. Wu J, Zeng T, Wu X, Gao Q, Zhai W and Ding Z: Ether à go-go 1 silencing in combination with TRAIL overexpression has synergistic antitumor effects on osteosarcoma. Cancer Biother Radiopharm 28: 65-70, 2012.
7. Mitsiades N, Poulaki V, Mitsiades C and Tsokos M: Ewing's sarcoma family tumors are sensitive to tumor necrosis factor-related apoptosis-inducing ligand and express death receptor 4 and death receptor 5. Cancer Res 61: 2704-2712, 2001.

8. Zheng SJ, Wang P, Tsabary G and Chen YH: Critical roles of TRAIL in hepatic cell death and hepatic inflammation. J Clin Invest 113: 58-64, 2004.

9. Miao J, Wu S, Peng Z, Tania M and Zhang C: MicroRNAs in osteosarcoma: diagnostic and therapeutic aspects. Tumour Biol 34: 2093-2098, 2013.

10. He C, Xiong J, Xu X, et al: Functional elucidation of MiR-34 in osteosarcoma cells and primary tumor samples. Biochem Biophys Res Commun 388: 35-40, 2009.

11. Ma L, Liu J, Shen J, et al: Expression of miR-122 mediated by adenoviral vector induces apoptosis and cell cycle arrest of cancer cells. Cancer Biol Ther 9: 554-561, 2010.

12. Liu J, Ma L, Li C, Zhang Z, Yang G and Zhang W: Tumor-targeting TRAIL expression mediated by miRNA response elements suppressed growth of uveal melanoma cells. Mol Oncol 7: 1043-1055, 2013.

13. Bo Y, Guo G and Yao W: MiRNA-mediated tumor specific delivery of TRAIL reduced glioma growth. J Neurooncol 112: 27-37, 2013.

14. Zhao Y, Li Y, Wang L, et al: microRNA response elements-regulated TRAIL expression shows specific survival-suppressing activity on bladder cancer. J Exp Clin Cancer Res 32: 10, 2013

15. Wang B,Liu J, Ma LN, et al: Chimeric 5/35 adenovirus-mediated Dickkopf-1 overexpression suppressed tumorigenicity of CD $44\left(^{+}\right)$gastric cancer cells via attenuating Wnt signaling. J Gastroenterol 48: 798-808, 2013.

16. Lou W, Chen Q, Ma L, et al: Oncolytic adenovirus co-expressing miRNA-34a and IL-24 induces superior antitumor activity in experimental tumor model. J Mol Med (Berl) 91: 715-725, 2013.

17. He X, Liu J, Yang C, et al: 5/35 fiber-modified conditionally replicative adenovirus armed with p53 shows increased tumor-suppressing capacity to breast cancer cells. Hum Gene Ther 22: 283-292, 2011. 